Methods We developed a murine monoclonal antibody to tissue bound $\mathrm{C} 3 \mathrm{~d}(\mathrm{mAb} 3 \mathrm{~d} 29)$ that targets tissue-bound $\mathrm{C} 3 \mathrm{~d}$ when injected in vivo. We stained biopsy tissue from patients with active $\mathrm{LN}$ to confirm that $3 \mathrm{~d} 29$ detects tissue $\mathrm{C} 3 \mathrm{~d}$ in human disease. We also analyzed biopsy reports from 76 patients with $\mathrm{LN}$ to assess whether $\mathrm{C} 3$ deposits correlate with disease activity.

We next radiolabeled mAb $3 \mathrm{~d} 29$ with ${ }^{124} \mathrm{I}$ and injected $16-$ week-old MRL/lpr mice (a model of lupus-like disease) or wild-type mice intravenously with $20 \mu \mathrm{g}$ of protein $(120 \mu \mathrm{Ci})$. The mice underwent whole body PET imaging using a Siemens Inveon microPET/CT scanner at 4, 24, 48, 72, and 96 hours post injection. After the mice were sacrificed, organs were weighed and counted in a gamma counter along with a known amount of the radioactive injectate to determine the biodistribution of the antibody.

Results By immunostaining, mAb 3d29 detects glomerular C3d in kidney samples from MRL/lpr mice and humans with LN. In patients with $\mathrm{LN}$, the abundance of glomerular C3 fragments $(0-3+)$ was highest in those patients with proliferative disease and with high activity scores.

${ }^{124}$ I-PET with $\mathrm{mAb} 3 \mathrm{~d} 29$ revealed rapid tracer uptake in the kidneys of MRL/lpr mice in the first hours after injection compared to controls, with retention up to 96 hours (figure $1)$. At the end of the study (144 hours), there was still a trend towards greater antibody in kidneys of MRL/lpr mice than controls $(4.93 \pm 1.42 \%$ vs $1.77 \pm 0.17$ percentage of injected radioactivity per gram of tissue, $\mathrm{P}=0.07$ )

Conclusion Glomerular C3 deposition is an important marker of disease activity in LN. C3d-imaging can be used to noninvasively detect inflammation in the kidneys of patients with LN.

\section{THE LOCALIZATION OF NOVEL MACROPHAGE SUBSETS IN CLASS III AND IV LUPUS NEPHRITIS KIDNEY SECTIONS}

\begin{abstract}
1, ${ }^{2}$ Paul J Hoover*, ${ }^{1}$ Michael Peters, ${ }^{3}$ Jeff Hodgin, ${ }^{1}$ Tony Jones, ${ }^{1} J o n$ Chen, ${ }^{4}$ Anne Davidson, ${ }^{1}$ Nir Hacohen. ${ }^{1}$ Broad Institute of MIT and Harvard, Cambridge MA, USA; ${ }^{2}$ Division of Rheumatology, Immunology, Department of Medicine, Brigham and Women's Hospital, Harvard Medical School, Boston MA, USA; ${ }^{3}$ Department of Pathology, Michigan Medicine, University of Michigan, USA; ${ }^{4}$ Feinstein Institutes for Medical Research, Manhasset NY, USA
\end{abstract}

\subsection{6/lupus-2021-lupus21 century.27}

Background Macrophage infiltration in lupus nephritis is associated with fibrosis and kidney damage. Prior histologic studies lacked the specificity of single-cell RNA sequencing (scRNAseq) for macrophage classification, so it was impossible to determine how the spatial organization of each subset related to kidney remodeling. Our recent scRNA-seq of macrophages has defined 3 novel subsets enriched in lupus nephritis over healthy kidneys: 'inflammatory' macrophages likely enter lupus kidneys from blood and shift to 'phagocytic' and 'reparativelike' states. Here, we mapped their positions in kidney sections from 20 different lupus nephritis patients using subsetspecific transcripts from our scRNA-seq data to reveal new macrophage spatial phenotypes.

Methods We collected and sectioned archived FFPE class III or IV index lupus nephritis kidney biopsies from Brigham and Women's Hospital. After standard FFPE antigen retrieval, we used commercial RNA probes against 2-3 highly specific genes based on scRNA-seq to stain each novel subset (inflammatory CSF1R+/CD300E+/CD36-, phagocytic CSF1R+/CD300E+/
CD36+; reparative CSF1R+/RNASE1+), and probes against non-mammalian genes as a negative control. We identified our macrophage subsets based on the presence of probes above the background and within 3 microns of the DAPI-stained nucleus as a cell boundary estimate. For spatial mapping, we transferred annotated histologic features from an adjacent H\&E section to sections stained for cells. For cell distance and density measurements we used HALO (Indica Labs).

Results Our in situ staining approach confirmed the presence of the novel inflammatory, phagocytic, and reparative macrophages discovered by scRNA-seq in class III and IV lupus nephritis kidney sections. Most inflammatory and phagocytic macrophages were localized to positions inside glomeruli while a smaller proportion in the tubulointerstitium formed a gradient toward the glomerular borders. Reparative macrophages were the most abundant macrophage subset in situ and were mostly in the tubulointerstitium arranged as a gradient toward glomerular borders. The abundance of reparative macrophages inside glomeruli varied across patients.

Conclusions Macrophage subsets were spatially localized to and around the glomerulus in lupus nephritis kidney sections. Inflammatory and phagocytic macrophage subsets were mostly inside the glomerulus, suggesting that glomerular factors supported their recruitment from blood and in situ differentiation. The most abundant subset - reparative macrophages were localized to the tubulointerstitial space and arranged in a gradient toward glomerular borders, indicating a chemical attraction to nephritic glomeruli and the presence of factors that promote reparative differentiation. Interestingly, the abundance of reparative macrophages inside glomeruli varied considerably across patients, raising the possibility that interpatient variability reflects differences in kidney function that we are now testing in an expanded cohort.

Acknowledgments Rheumatology Research Foundation, Lupus Research Alliance, Lupus Foundation of America

\section{B-CELL INTERFERON- $\beta$ CORRELATES WITH LUPUS NEPHRITIS IN SYSTEMIC LUPUS ERYTHEMATOSUS}

\begin{abstract}
1,2,3 Fatima Alduraibi, ${ }^{4}$ Huma Fatima, ${ }^{5}$ Jennie A Hamilton, ${ }^{1}$ Walter Winn Chatham* ${ }^{*}{ }^{1}$ HuiChen Hsu, ${ }^{1,2}$ John D Mountz. 'Department of Medicine, the University of Alabama at Birmingham, Birmingham, AL, USA; ${ }^{2}$ Birmingham VA Medical Center, Birmingham, AL, USA; ${ }^{3}$ Department of Medicine, King Faisal Specialist Hospital and Research Center, Riyadh, $S A ;{ }^{4}$ Department of Pathology, the University of Alabama at Birmingham, Birmingham, AL, USA; ${ }^{5}$ Department of Medicine, the University of Tennessee at Memphis, Memphis, TN, USA
\end{abstract}

\subsection{6/lupus-2021-lupus21 century.28}

Background Early diagnosis of lupus nephritis (LN) can be challenging since some patients do not exhibit overt clinical manifestations until advanced stages. B cell interferon-beta (IFN $\beta$ ) correlates with development of B cell autoreactive phenotype. The objective of the present study is to determine if elevated IFN $\beta$ in circulating B cells can be a useful indicator for the development of more severe histopathologic features of LN.

Methods Flow cytometry was used to quantitate intracellular IFN $\beta$ in naive $(\operatorname{IgD}+\mathrm{CD} 27-) \mathrm{CD} 19+\mathrm{B}$-cells in the peripheral blood mononuclear cells (PBMCs) of a cross-sectional cohort $(\mathrm{N}=80)$ of patients with systemic lupus erythematosus (SLE), 33 of whom had lupus nephritis. Serologic and clinical manifestations of LN included anti-DNA, anti-Sm, C3, C4, and urine protein/creatinine ratio were determined. The correlation of B-cell IFN $\beta$ with lupus nephritis classification and 